\title{
CrystEngComm
}

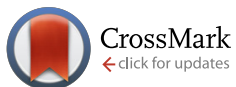

Cite this: CrystEngComm, 2017, 19, 876

Received 9th October 2016, Accepted 29th December 2016

DOI: $10.1039 / c 6 c e 02149 b$

www.rsc.org/crystengcomm

\section{Non-classical growth of water-redispersible spheroidal gold nanoparticles assisted by leonardite humate $\dagger$}

\author{
Alexander Yu. Polyakov, ${ }^{a}$ Vasily A. Lebedev, ${ }^{\text {ac }}$ Evgeny A. Shirshin, ${ }^{\text {b }}$ \\ Artem M. Rumyantsev, ${ }^{b}$ Alexander B. Volikov, ${ }^{c}$ Alexander Zherebker, ${ }^{c}$ \\ Alexey V. Garshev, ${ }^{\text {acd }}$ Eugene A. Goodilin ${ }^{\text {ac }}$ and Irina V. Perminova*c
}

\begin{abstract}
The growth of gold nanoparticles (AuNPs) assisted by humate - a natural hyperbranched polyelectrolyte was studied using in situ and ex situ techniques. The conditions for formation of almost monodisperse gold nanospheres of $13 \pm 3 \mathrm{~nm}$ diameter with pronounced plasmonic properties were defined. A striking similarity was found between humate- and citrate-mediated growth of AuNPs: the formation of gold nanospheres involved rapid nucleation of gold seeds, slow growth within the intermediate agglomerates (visible as nanoworms after drying on a TEM grid), and rapid peptization into the final gold nanospheres. Both humate and citrate syntheses produced ultrastable gold sols with pronounced plasmonic properties. The substantial difference was slower kinetics of humate synthesis, 240 min versus 15 min for citrate, and the water redispersible properties of the humate-capped gold nanospheres after freeze drying, which was not seen with the citrate AuNPs. Theoretical calculations revealed a leading role of steric factors in the formation of intermediate aggregates of capped AuNPs at the stage of their slow growth in the case of both citrate and humate. We suggested that it was the polyelectrolyte nature of humate which enabled the waterredispersibility of humate- versus citrate-capped gold nanoparticles.
\end{abstract}

\section{Introduction}

Gold nanoparticles (AuNPs) have attracted substantial attention due to their size- and shape-tunable optoelectronic properties and catalytic and biological stability, which offer a wide range of applications in nano- and biomedical technologies. ${ }^{1,2}$ AuNPs are frequently produced using toxic reducing agents, solvents, or stabilizers for tailoring unique

\footnotetext{
${ }^{a}$ Department of Materials Science, Lomonosov Moscow State University, Leninskie Gory 1-73, 199991 Moscow, Russia

${ }^{b}$ Department of Physics, Lomonosov Moscow State University, Leninskie Giry 1-2, 119991 Moscow, Russia

${ }^{c}$ Department of Chemistry, Lomonosov Moscow State University, Leninskie Gory 1-3, 119991 Moscow, Russia. E-mail: iperm@org.chem.msu.ru;

Fax: +7 49593955 46; Tel: +7 4959395546

${ }^{d}$ Baikov Institute of Metallurgy and Materials Science of RAS, Leninskiy Prospect 49, 199991 Moscow, Russia

$\dagger$ Electronic supplementary information (ESI) available: Additional information about real-time UV-vis spectroscopy, fluorescence spectroscopy, Fourier transform ion cyclotron resonance mass spectrometry and theoretical calculations; HRTEM and XRD analysis of the obtained AuNPs; figures demonstrating the colloidal stability of the prepared AuNP sols; considerations on the non-redispersible behavior of citrate-capped AuNPs. See DOI: 10.1039/ c6ce02149b
}

properties. ${ }^{3-9}$ As a result, a whole suite of greener synthetic routes has been developed, which implies the use of ecofriendly natural compounds and biopolymers. ${ }^{10-16}$ Among these are natural polyelectrolytes - humic substances (HS) which have a very good safety profile and abundant resources (e.g., lignites). ${ }^{17}$ The presence of multiple carboxyl, hydroxyl, and carbonyl groups results in remarkable macroligand, surface-active and redox properties of HS. ${ }^{18-22}$ They are used as capping and stabilizing agents for engineered nanoparticles including AuNPs. ${ }^{23-29}$ The use of different fractions of soil HS enabled fabrication of anisotropic gold nanoplates with enhanced optical properties. ${ }^{30}$ To improve control over the size and shape of optically active AuNPs, synthetic HS were employed as reducing and capping agents. ${ }^{31}$ However, the use of humates for the preparation of monodisperse spheroidal gold nanoparticles similar in properties to citrateAuNPs remains an unresolved problem.

In our previous studies we demonstrated how humic polyanions derived from leonardite could be efficiently used to control the growth (sizes) and morphology of iron oxohydroxide nanoparticles. ${ }^{32,33}$ We have also shown the excellent colloidal stability and biocompatibility of humate-capped NPs. ${ }^{34,35}$ 
In this work, we used leonardite-derived humate as a capping agent to achieve control over the morphology, size distribution, plasmonic properties, and water redispersibility of AuNPs. Firstly, we defined the conditions for humatemediated synthesis of spheroidal AuNPs with a narrow size distribution and enhanced optical properties; secondly, we studied the growth of spheroidal humate AuNPs as compared to citrate AuNPs; thirdly, we surmised the mechanisms which underlie the ultrastability and water-redispersibility of AuNPs capped with polyelectrolytic humate as opposed to low molecular weight citrate.

\section{Experimental}

\section{Gold nanoparticle synthesis}

Tetrachloroauric(III) acid was from Sigma-Aldrich (G4022 product, ACS reagent, $\geq 49.0 \% \mathrm{Au}$ basis). All other reagents were of analytical grade. All solutions were prepared using distilled water (Milli-Q RG, Millipore). Glassware and magnetic stirring bars were pre-washed with aqua regia (a mixture of hydrochloric $(\mathrm{HCl})$ and nitric $\left(\mathrm{HNO}_{3}\right)$ acids at a molar ratio of $3: 1$ ).

Commercially available potassium humate from leonardite (a highly oxidized variety of lignites) (CHP) was used in our studies. It was characterized using elemental and functional group analyses, ${ }^{13} \mathrm{C}$ NMR spectroscopy, and size exclusion chromatography (SEC). The $\mathrm{H} / \mathrm{C}$ and $\mathrm{O} / \mathrm{C}$ atomic ratios were 0.84 and 0.38 , respectively, showing the highly aromatic character of the humic material used. This was also confirmed by the ${ }^{13} \mathrm{C}$ NMR data on carbon distribution among the structural fragments (in \% of the total $\mathrm{C}$ ): 15\% aliphatic $\mathrm{C}, 7 \%$ aliphatic $O$-substituted C, 47\% aromatic C, 12\% aromatic $O$-substituted C, 13\% carboxylic and ester C, 5\% ketone C. The functional group analysis yielded a total acidity of $5.3 \pm$ $0.9 \mathrm{mmol} \mathrm{g}^{-1}$ and carboxylic acidity of $2.9 \pm 0.5 \mathrm{mmol} \mathrm{g}^{-1}$. The SEC-determined weight-average molecular weight $\left(M_{\mathrm{w}}\right)$ was $10.0 \pm 0.2 \mathrm{kDa}$, and the number-average molecular weight $\left(M_{\mathrm{n}}\right)$ was $5.4 \pm 0.1 \mathrm{kDa}$.

An amount of the CHP sample was dissolved in MilliQ water and centrifuged to prepare a HS stock solution with a concentration of $212 \mathrm{mg} \mathrm{L}^{-1}$.

The synthesis of AuNPs was carried out under $\mathrm{pH}$ control and the $\mathrm{pH}$ value was monitored and logged throughout the reaction using Expert-001 pH monitoring system and Expert$00 x$ software (Econics-Expert Ltd., Russia). For this purpose, a working solution of CHP with a concentration of $85 \mathrm{mg} \mathrm{L}^{-1}$ was placed in a foil-wrapped glass vessel and heated up to 70 ${ }^{\circ} \mathrm{C}$. $\mathrm{HAuCl}_{4}$ solution was added under vigorous stirring to give $5 \times 10^{-4} \mathrm{M} \mathrm{HAuCl}_{4}$. Immediately after $\mathrm{HAuCl}_{4}$ addition, the $\mathrm{pH}$ was adjusted to 7.0 with $0.1 \mathrm{M} \mathrm{KOH}$ (no further $\mathrm{pH}$ adjustment was made). The reaction mixture was stirred continuously for 4 hours at $70{ }^{\circ} \mathrm{C}$. The same synthesis procedure was carried out at initial $\mathrm{pH}$ values of 3,5 , and 9. The samples were designated as Au-HS- $x$, where $x$ is the initial $\mathrm{pH}$ value of the synthesis. A working humate solution $(85 \mathrm{mg}$ $\mathrm{L}^{-1}$ ) without $\mathrm{HAuCl}_{4}$ addition was used in a blank experi- ment. It was brought to $70{ }^{\circ} \mathrm{C}$ and stirred for 4 hours at $\mathrm{pH}$ 7.0.

An additional synthesis of humate-AuNPs at $\mathrm{pH} 7.0$ was conducted under nitrogen atmosphere. The working CHP solution was purged by nitrogen gas for an hour until $\mathrm{HAuCl}_{4}$ was added. Then, the $\mathrm{pH}$ value was adjusted to 7.0, and the reaction mixture was vigorously stirred for 4 hours at $70{ }^{\circ} \mathrm{C}$. The sample was designated as Au-HS-7- $\mathrm{N}_{2}$.

The synthesis of citrate-AuNPs is described in the ESI. $\dagger$

Dry powders of humate- and citrate-stabilized AuNPs were prepared by freeze-drying in a Labconco FreeZone freeze dry system $\left(-50{ }^{\circ} \mathrm{C}, 0.03\right.$ mbar pressure). For redispersion, a dried AuNP sample was dissolved in $0.1 \mathrm{M} \mathrm{KOH}$ under sonication $(10 \mathrm{~min})$; then the $\mathrm{pH}$ was adjusted to 7 using $0.1 \mathrm{M}$ HCl.

\section{Analytical methods}

UV-visible absorption spectra were recorded using a PerkinElmer Lambda 950 spectrophotometer (transmission geometry, wavelength range of $200-1000 \mathrm{~nm}, 1 \mathrm{~nm}$ step). The plasmonic spectra of AuNPs were collected immediately after preparation and after storing in the dark for two months and two years. A custom-built set-up was used for real-time recording of UV-vis absorption spectra, as described in the ESI. $†$

The NP morphology was analyzed using a Carl Zeiss Libra 200 MC transmission electron microscope operating at 200 $\mathrm{kV}$. The reaction mixture was dripped on lacey-carbon copper grids (SPI, USA) at different time-delays after $\mathrm{HAuCl}_{4}$ addition, blotted and dried. Images were processed using opensource Gwyddion software, ${ }^{36}$ and particle size distributions were calculated using $300+$ particles of each sample.

Fluorescence spectra were recorded using a Fuoromax-4 spectrofluorometer (Horiba Jobin Yvon) at $266 \mathrm{~nm}$ excitation; excitation and emission slit widths were set to $5 \mathrm{~nm}$. For fluorescence measurements, $25 \mathrm{ml}$ of reaction mixtures were taken and dissolved in $3 \mathrm{ml}$ of distilled water.

Fourier transform ion cyclotron resonance mass spectrometry (FTICR MS) analysis was conducted using facilities of the Emanuel Institute of Biochemical Physics of RAS (Moscow). The analytical conditions and data interpretation are described in the ESI. $\dagger$

\section{Results and discussion}

\section{Impact of $\mathrm{pH}$ on the size and shape of the humate-AuNPs}

We have carried out humate-mediated syntheses of AuNPs under $\mathrm{pH}$ control (initial $\mathrm{pH}$ values were adjusted to 3, 5, 7, and 9) to define the conditions which favor the size and shape homogeneity of AuNPs. The change in $\mathrm{pH}$ altered the macroligand and redox properties of humic polyanions. At $\mathrm{pH} 3$, the major portion of acidic groups is protonated, which minimizes the charge of humic polyanions and maximizes their coiling. An increase in $\mathrm{pH}$ brings about a growth in charge density due to deprotonation of carboxyl groups, which reaches $100 \%$ at $\mathrm{pH}$ 7. This $\mathrm{pH}$ also favors the maximum oxidizing ability of phenolic groups. ${ }^{37}$ It should be 
noted that speciation of $\mathrm{Au}^{\mathrm{III}}$ is also governed by $\mathrm{pH}$ in aqueous chloride-containing solutions: an increase in $\mathrm{pH}$ yields more hydrolyzed auric species with lesser chemical reactivities. $^{38,39}$

Fig. 1 demonstrates the UV-vis absorption spectra, sizes and morphologies of the humate-AuNPs synthesized at different $\mathrm{pH}$ values. For comparison, the corresponding data for citrate-AuNPs are shown. ${ }^{40}$ The closest similarity with citrateAuNPs with respect to size, shape and optical properties (Fig. 1b) was seen for AuNPs synthesized at $\mathrm{pH} 7$ (Fig. 1c and d): they had the smallest size (13 $\pm 3 \mathrm{~nm})$, a spheroidal shape, and the narrowest SPR peak located at 524 $\mathrm{nm}$. All other $\mathrm{pH}$ values (3, 5 and 9) brought about the formation of irregularly shaped AuNPs (Fig. 1e-g). The HRTEM image of a single AuNP reveals a crystalline structure whose interplanar distances $(2.35 \AA)$ correspond to that of fcc metallic gold (Fig. 1d). HRTEM allowed visualization of numerous dislocations and twin boundaries in the synthesized AuNPs (Fig. 1d and S1†). The particle phase compositions were determined using XRD (Fig. S2 $\dagger$ ).

The appearance of ellipsoidal or rod-like AuNPs at $\mathrm{pH} 5$ and 9 led to a red shift of the SPR peak and caused its broadening (Fig. 1a, e and f). The shoulder at $625 \mathrm{~nm}$ was observed for the Au-HS-3 sample, which could be explained by the formation of triangular/hexagonal gold nanoprisms (Fig. 1g).

All Au-HS sols obtained in this study showed high colloidal stability: the position and intensity of the SPR peak did not change over two years (Fig. S4 $\dagger$ ). This corroborates with the reported findings on HS as an effective reducing and stabilizing agent for the production of gold sols. ${ }^{29-31}$ However, only AuNPs synthesized at pH 7 had a spheroidal shape and narrow size distribution closely resembling those of citrate AuNPs. ${ }^{40,41}$ To understand whether the resemblance of AuHS-7 and citrate-AuNPs was a "happy coincidence", or both processes are governed by similar mechanisms, we have conducted detailed kinetic studies on humate-AuNP formation at pH 7 and compared them with the citrate synthesis.

\section{Kinetics and chemistry of AuNP formation assisted by leonardite humate at $\mathrm{pH} 7$}

The TEM images of humate-AuNPs in the course of their formation at $\mathrm{pH} 7$ were obtained $e x$ situ and shown in Fig. 2a-c. The UV-vis spectra were recorded in situ and processed to calculate the maximum wavelength, FWHM, and area of the SPR peak of the humate-AuNPs. The corresponding data are

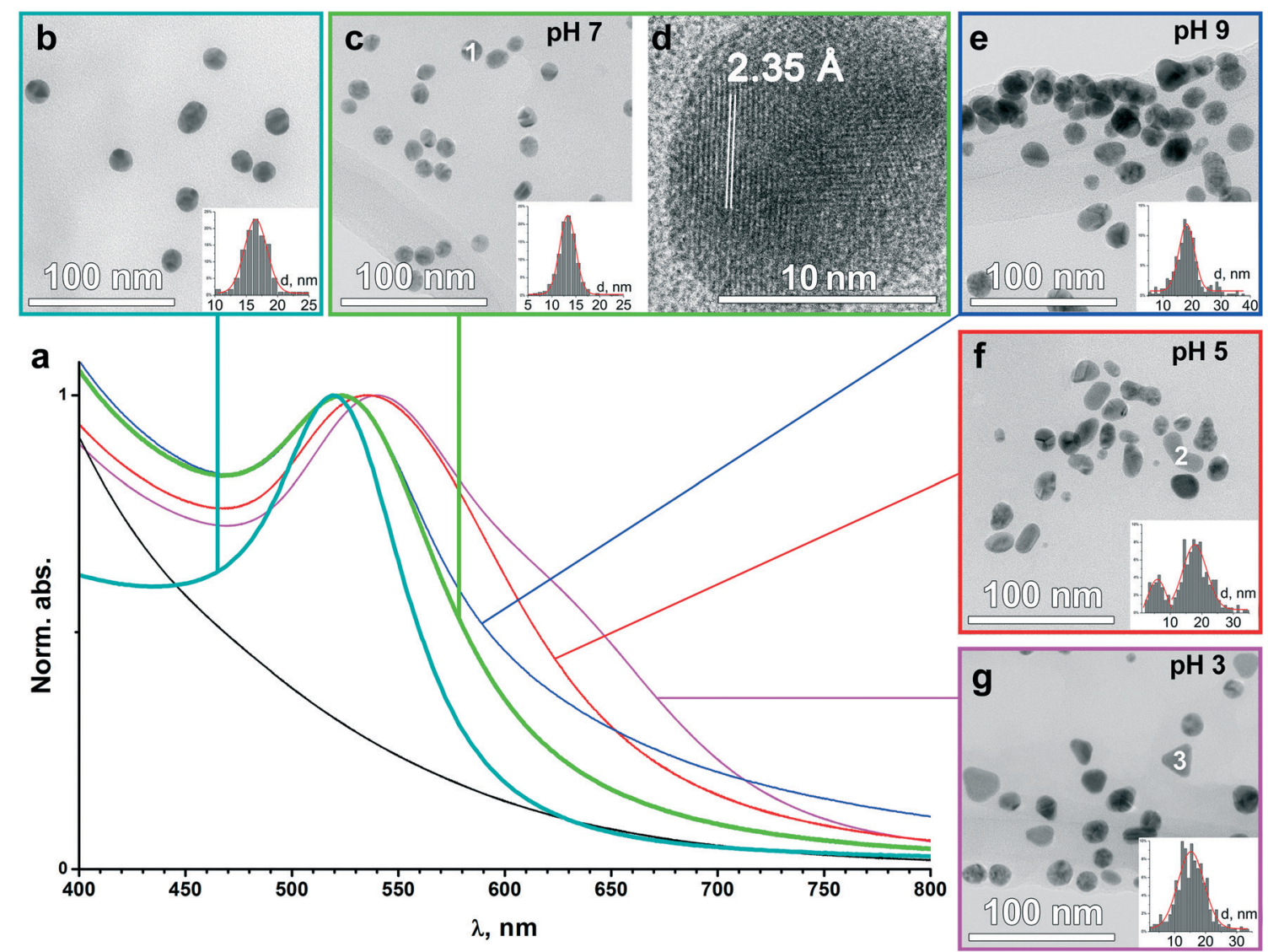

Fig. 1 Humate-AuNPs obtained at different initial pH values. (a) UV-vis absorption spectra: black - initial HS solution, dark cyan - citrate-AuNPs, green - Au-HS-7, blue - Au-HS-9, red - Au-HS-5, magenta - Au-HS-3. TEM micrographs: (b) citrate-AuNPs $\left(0.25 \mathrm{mM}^{\mathrm{HAuCl}} \mathrm{H}_{4}+1.75 \mathrm{mM}\right.$ $\mathrm{Na}_{3} \mathrm{C}_{6} \mathrm{H}_{5} \mathrm{O}_{7}, 100{ }^{\circ} \mathrm{C}$; ; (c) Au-HS-7; (d) magnified view of a single Au-HS-7 particle; the atomic interplanar distance of metallic gold is highlighted; (e) Au-HS-9; (f) Au-HS-5; (g) Au-HS-3. Three major types of humate-AuNP morphologies are shown with numbers: 1 - nanospheres (c); 2 - irregularly shaped NPs (f); 3 - prismatic nanoplatelets (g). Full-size graphs of particle size distributions for all Au-HS samples are given in the ESI $\nmid$ (Fig. S3). 

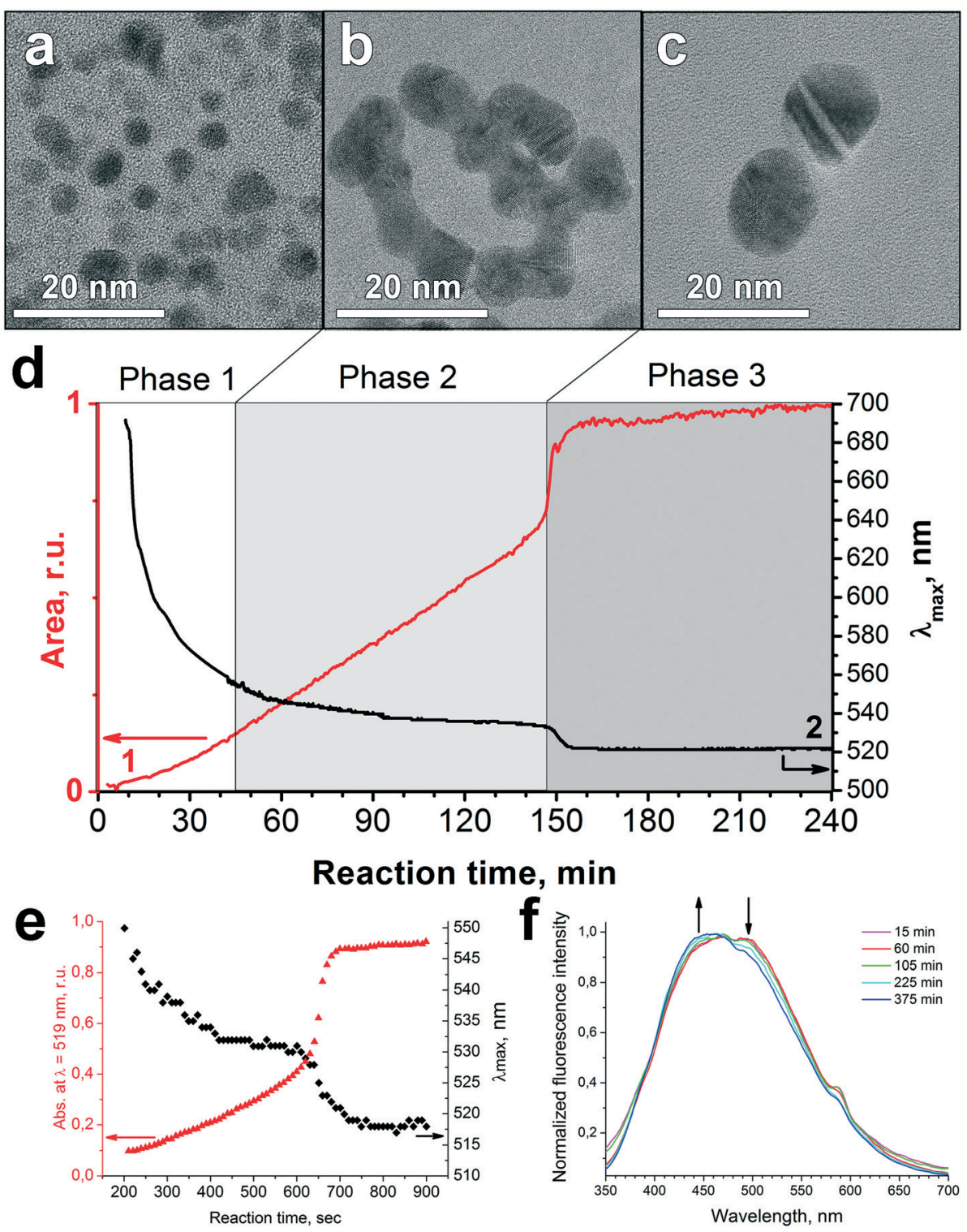

Fig. 2 Evolution of humate-AuNP formation at pH 7: TEM images of (a) AuNP seeds at the initial step, (b) worm-like intermediate aggregates, and (c) final spheroidal NPs. (d) Time evolution of SPR peak parameters ( $1-$ area, $2-\lambda_{\max }$ ). (e) Time evolution of the SPR band during the reaction of $2.53 \times 10^{-4} \mathrm{M} \mathrm{HAuCl}_{4}$ with $1.70 \times 10^{-3} \mathrm{M}$ citrate at $100{ }^{\circ} \mathrm{C}$ (numerical data adopted from ref. 42). (f) Change in fluorescence spectra of HS over reaction time.

plotted in Fig. 2d and $55 \dagger$ (the latter includes FWHM and $\mathrm{pH}$ curves recorded during the synthesis). Similar SPR data reported for the citrate-AuNPs are given in Fig. 2e for comparison. The fluorescence spectra of the Au-HS reaction mixture were recorded over the entire reaction time (Fig. 2f) to monitor changes in humate optical properties.

Evolution of the SPR band during the reaction of $\mathrm{HAuCl}_{4}$ with potassium humate revealed three stages in humateAuNP formation as indicated in Fig. 2d. The first phase, nucleation, started immediately after $\mathrm{HAuCl}_{4}$ addition to humate solution with the formation of numerous gold seeds of $3.0 \pm 0.5 \mathrm{~nm}$ diameter (Fig. 3a). At this time some large dendritic aggregates (up to $150 \mathrm{~nm}$ ) were formed as well (Fig. $\mathrm{S} 6 \dagger$ ), which could originate from local supersaturations upon the addition of concentrated $\mathrm{HAuCl}_{4}$. These aggregates might be responsible for the low-intensity SPR signal at 600-700 $\mathrm{nm}$ at the first minutes of the reaction. These large structures were not observed at later reaction times, probably due to dissolution or sedimentation.

The second phase was characterized by the appearance of a network of worm-like AuNP-structures (Fig. 2b). The latter seem to be artifacts of drying on the TEM grid, while in the reaction mixture the $5 \pm 2 \mathrm{~nm}$ AuNPs did not merge within these agglomerates. However, their close proximity gave rise to coupling of plasmonic oscillations, which explains the position of the SPR peak at ca. $550 \mathrm{~nm}$ (Fig. 2d), while the expected SPR wavelength for $5 \mathrm{~nm}$ gold nanospheres in water is $c a .517 \mathrm{~nm}^{43}$ At this phase the SPR peak shifted slowly from 550 to $535 \mathrm{~nm}$ and its area increased steadily indicating the growth of humate-AuNPs over $145 \mathrm{~min}$ (Fig. 2d). 

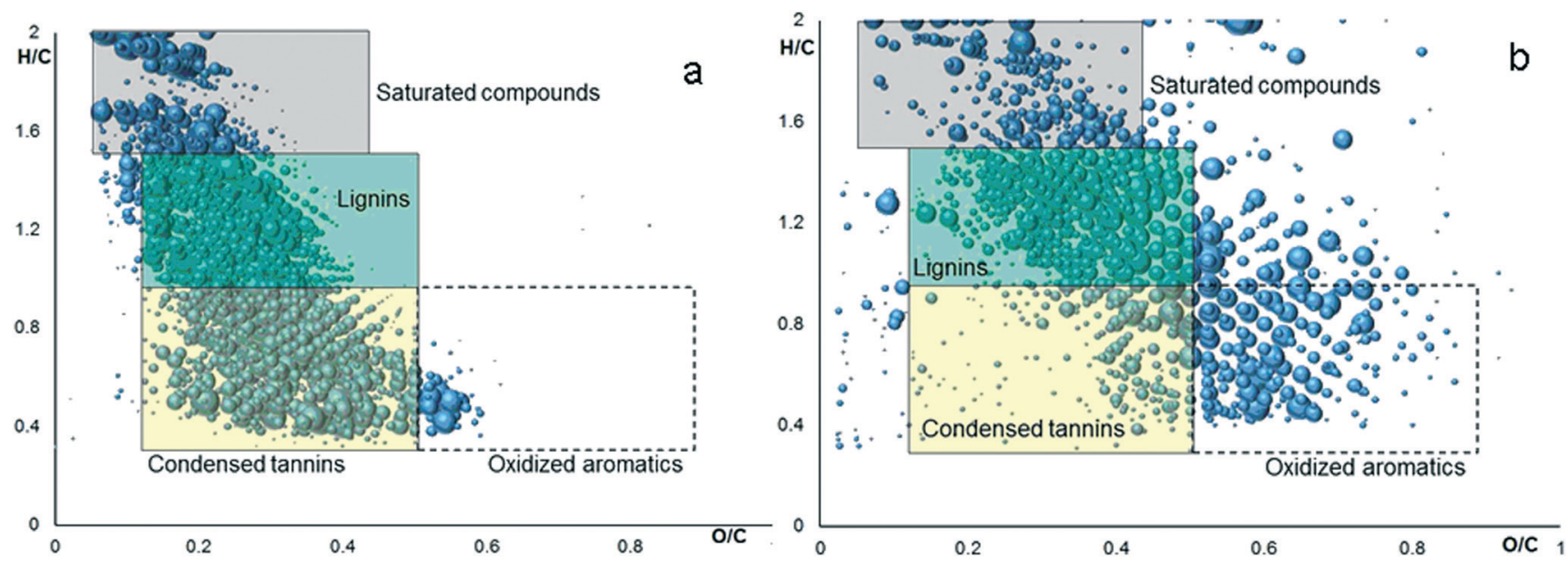

Fig. 3 Van Krevelen diagrams plotted from FTICR-MS identification of potassium humate which was obtained before (a) and after (b) its reaction with $\mathrm{HAuCl}_{4}$ at pH 7 (AuHS-7).

After reaching $145 \mathrm{~min}$, the SPR peak moved suddenly to $524 \mathrm{~nm}$, which was followed by its significant narrowing and $30 \%$ increase in intensity (Phase 3). TEM imaging suggests that such abrupt and rapid changes were caused by peptization of the worm-like agglomerates into nearly monodisperse spheroidal AuNPs with a mean diameter of $13 \pm 3 \mathrm{~nm}$ (Fig. 2c).

The evolution of plasmonic spectra of humate-AuNPs resembles closely the citrate-mediated synthesis shown in Fig. 2e. ${ }^{42}$ Both syntheses are characterized by a steady blue shift of $\lambda_{\max }$ over the reaction time until an abrupt change takes place in both the SPR wavelength and its intensity. This suggests that both syntheses may have similar particle growth mechanisms. Similarly to citrate, humate plays a role of acid-base buffer at $\mathrm{pH} 7$, producing multidentate polyanions due to deprotonation of carboxyl groups. These carboxylates are capable of ligand exchange with $\left[\mathrm{AuCl}_{4-n} \mathrm{OH}_{n}\right]^{-}$ species stabilizing them in solution. Simultaneously, the phenolic groups of humic macroligands act as reductants for $\left[\mathrm{AuCl}_{4}\right]^{-}$ions. In contrast to citrate, the oxidation of phenolic groups is a two stage reversible process yielding the phenoxyl radical. The rate of oxidation is much slower as compared to that of citrate due to the formation of stable semiquinone radicals. Ultimately, further oxidation of phenoxyl radicals would lead to irreversible cleavage of aromatic rings followed by formation of dibasic acids.

We used fluorescence spectroscopy for monitoring humate transformation during the growth of AuNPs. The normalized spectra are shown in Fig. 2f. They did not reveal any change in the humate fluorescence spectral band shape $\left(\lambda_{\text {exc }}=266\right.$ $\mathrm{nm}$ ) during the first 60 minutes of reaction time. After that a gradual decrease was observed in the relative intensity of the red edge fluorescence. These changes could not be attributed to a $\mathrm{pH}$ increase during the synthesis, which we checked by conducting fluorescence measurements at different $\mathrm{pH}$ values (Fig. S7†). Instead, they could be related to partial degradation of the conjugated system of fluorophores contributing to the red edge of HS fluorescence. ${ }^{44}$ Direct evidence of partial HS oxidation was obtained using FTICR MS analysis of the humate before and after its reaction with $\mathrm{HAuCl}_{4}$ (Fig. 3).

Fig. 3 shows FTICR MS identifications plotted in Van Krevelen diagrams, which establish the relationships between $\mathrm{H} / \mathrm{C}$ and $\mathrm{O} / \mathrm{C}$ atomic ratios for molecular constituents of the analyzed mixture. Color mapping relates the diagram areas to predominant chemical classes observed in these regions. This enables comparison of the molecular composition of the CHP before and after reaction with $\mathrm{HAuCl}_{4}$ (Fig. 3a and b, respectively). Thus, the contribution of condensed phenolic constituents, which populate the yellow field of the diagram, decreased from $46 \%$ to $25 \%$ after reaction, whereas the content of oxygen rich aromatic compounds (white field) increased from $2 \%$ to $18 \%$. This is indicative of oxidative transformation of humic phenolic constituents. The content of other humic components underwent very little change. This provides for stability of the humic matrix over the entire reaction time. The high-resolution views, references, and details of FTICR MS experiments are given in the ESI. $\dagger$

The kinetic data allowed us to propose the following scheme of the three-phase growth of AuNPs mediated with humate which leads to the formation of stable sols of spherical AuNPs:

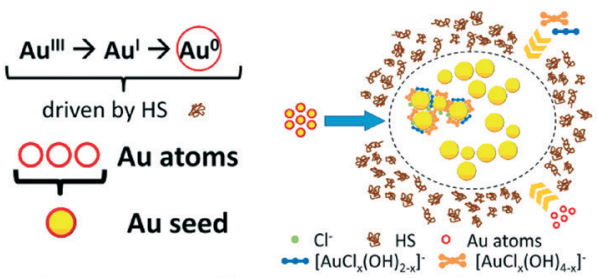

Phase 1: Seeding Phase 2: Slow Growth

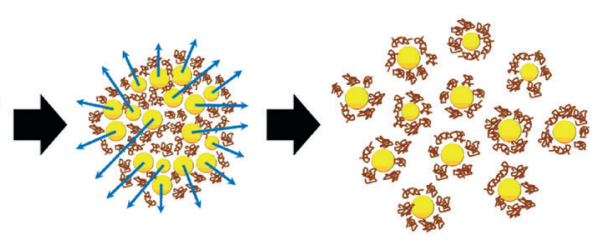

Phase 3: Fast Peptization 
The given scheme was further backed up by considerations of the underlying chemistry including the redox chemistry of $\mathrm{HS}$, and the reported data on the chemistry of $\mathrm{HAuCl}_{4}$ at $\mathrm{pH} 7 .^{38,39}$

At stage 1 , highly reactive species of $\left[\mathrm{AuCl}_{4}\right]^{-}$are reduced by oxidizing phenolic units of humate to $\mathrm{Au}^{0}$ or $\mathrm{Au}^{\mathrm{I}}$ which is accompanied by proton release causing a drop in the $\mathrm{pH}$ curve down to 5.8 within the first 15 min (Fig. S5†). At the same time the hydrolysis of $\left[\mathrm{AuCl}_{4}\right]^{-}$(also lowering the $\mathrm{pH}$ value) gives much less reactive species of $\left[\mathrm{AuCl}_{4-n}(\mathrm{OH})_{n}\right]^{-}$ while the formation of complexes with humate ions slows down new $\mathrm{Au}^{0}$ generation, turning the reaction to a surface growth mode.

At stage 2, the metallic gold surface of the formed seeds absorbs both $\mathrm{Au}^{\mathrm{III}}$ and $\mathrm{Au}^{\mathrm{I}}$ species and autocatalyses their reduction and $\mathrm{Au}^{\mathrm{I}}$ disproportionation. ${ }^{45}$ Complexation with oxidized HS can also facilitate $\mathrm{Au}^{\mathrm{I}}$ disproportionation similarly to dicarboxyl acetone in citrate-mediated synthesis. ${ }^{40,46}$ Over this time random flocculation of $\mathrm{Au}^{0}$ seeds and particles occurs, yielding intermediate agglomerates visualized by $e x$ situ TEM as worm-like structures (Fig. 2b).

At stage 3, peptization of AuNPs observed in the humatemediated system after 145 minutes is similar to that described by Biggs et $a l^{47}$ and Wall et $a .^{48}$ for citrate-gold surface interactions: they detected a change from attractive to repulsive interactions at the gold surface resulting from a gradual decrease of auric ion concentration and accumulation of citrate in the electrical double layer of the particles. In our system, formation of a large area of the gold surface with a positive charge was reflected in the $\mathrm{pH}$ curve as a slight decrease which could result from the sorption of $\mathrm{OH}^{-}$ anions (Fig. S5†).

Of interest is that the widely used citrate-mediated growth of gold nanospheres is also non-classical and involves agglomeration of the primary gold seeds and particle growth within the intermediate agglomerates. ${ }^{49}$ The appearance of intermediate worm-like structures on ex situ TEM images was reported elsewhere. ${ }^{39,50,51}$ At the same time, some SAXS studies did not detect formation of the aggregates during the citrate-mediated synthesis. ${ }^{52,53}$

It should be stressed that the non-classical growth of AuNPs seems to be feasible only within a certain " $\mathrm{pH}$ window" in the case of both citrate and humate. Ji et al. ${ }^{39}$ observed intermediate aggregation only in the $\mathrm{pH}$ range from 3.7 to 6.5. Our observations show higher $\mathrm{pH}$ values: from 6.5 to 8.0. We surmised that in the case of humate, the $\mathrm{pH}$ drop caused by $\mathrm{Au}(\mathrm{III})$ hydrolysis was compensated for by humate decarboxylation (Fig. S5†), which sustained the higher $\mathrm{pH}$ values. The humate decarboxylation might be triggered by oxidation of its alcoholic groups to $\beta$-keto acids by dissolved oxygen. ${ }^{54}$ We checked this hypothesis by conducting Au-HS-7 synthesis under nitrogen atmosphere. Indeed, in the absence of oxygen we observed a pH drop to 4.85 by the time of reaction completion (Fig. S9†), which yielded randomly shaped AuNPs (Fig. S10 $†$ ). The obtained results corroborate well with the findings on the important role of dissolved oxygen in re- dox reactions and particle growth processes. ${ }^{55}$ The remarkable impact of a $\mathrm{N}_{2}$ atmosphere was also reported for radiolytic synthesis of citrate AuNPs. ${ }^{56}$

Further, we undertook theoretical studies on gold colloidal stability during the humate synthesis to get deeper insight into the peptization mechanism of intermediate AuNP agglomerates.

\section{Theoretical calculations on the colloidal stability of AuNPs stabilized by humate and citrate}

Chow and Zukoski ${ }^{49}$ developed a model for gold sol formation in citrate-mediated synthesis incorporating colloidal stability. They suggested the formation of intermediate fluffy flocs where direct contact and irreversible aggregation of gold subunits are limited by a steric barrier produced by adsorbed ions. This is in accordance with in situ SAXS studies, ${ }^{51-53}$ which could not detect merged AuNP aggregates in a reaction mixture, leading the authors to conclude that the merging of NPs within dendrites, worm-like intermediates, etc., observed by ex situ TEM $^{39,50,51}$ is a result of a drying process. To define the factors providing for reversible AuNP flocculation and stabilization of intermediate aggregates with remaining interparticle separation, the pairwise particle interaction potential of AuNPs was calculated theoretically.

For this purpose, we considered the interaction of two identical charged spherical AuNPs with a radius $a$. Let $r$ be the distance between their centers; thus $h=r-2 a$ is the shortest distance between their surfaces. The total energy of interaction of colloidal NPs $\left(V_{\text {tot }}\right)$ expressed in $k_{\mathrm{B}} T$ units $\left(k_{\mathrm{B}}\right.$ and $T$ are the Boltzmann constant and absolute temperature, respectively) is composed of three terms:

$$
V_{\text {tot }}=V_{\mathrm{vdW}}+V_{\mathrm{el}-\mathrm{st}}+V_{\mathrm{solv}}
$$

In accordance with the classical DLVO theory, ${ }^{57}$ the first two terms correspond to van der Waals attraction and electrostatic repulsion, respectively, while the third one accounts for non-DLVO solvation forces. The attraction potential $\left(V_{\mathrm{vdw}}\right)$ is given by the Hamaker expression for spherical particles ${ }^{58}$ where we used the reported value of the gold/water/gold Hamaker constant $A=2.5 \times 10^{-19} \mathrm{~J}$ measured by AFM. ${ }^{59} \mathrm{We}$ used the Ohshima expression ${ }^{60-62}$ for calculation of electrostatic repulsion potential $\left(V_{\mathrm{el}-\mathrm{st}}\right)$, since the condition $a / r_{\mathrm{D}}<5$ was fulfilled. Here $a$ is the particle radius assumed to be 8.5 $\mathrm{nm}$ (estimated from our TEM results, Fig. 2), and $r_{\mathrm{D}}$ is the Debye radius equal to $1.8 \mathrm{~nm}$ for the ionic strength of the citrate-based reaction mixture of $2.5 \times 10^{-2} \mathrm{M}$. The Bjerrum length $l_{\mathrm{b}}$ was set to $0.6 \mathrm{~nm}$ because the syntheses were performed at $T=350 \mathrm{~K}$. At particle separations lower than a few nanometers, solvation forces may substantially influence interparticle interaction and play a key role in dispersion stabilization. We approximated the steric repulsion of soft adsorbed layers by a smooth repulsive potential $V_{\text {solv }}$ instead of the infinite vertical potential wall, which was used by Chou and Zukoski. ${ }^{49}$ This allowed us to get both primary and 
secondary minima at the interaction potential curve. Thus, the last term calculated in the framework of Derjaguin approximation $(h \ll a)^{57}$ reads

$$
V_{\text {solv }}=2 \pi \frac{B_{\mathrm{s}} a l}{k_{\mathrm{B}} T} \exp \left(\frac{h}{l}\right)
$$

with the decay length $l=0.5 \mathrm{~nm}$ and parameter $B_{\mathrm{s}}=22 \times$ $10^{-3} \mathrm{~J} \mathrm{~m}^{-2}$. Full mathematical expressions and corresponding assumptions are provided in the ESI. $\dagger$

Given that humate (or citrate) ions replace gold ions at the gold-water interface and cause an increase in the surface potential $\psi_{0}$, a model was developed for the qualitative explanation of how particle surface potential affects the dispersion stability. Fig. 4 shows a set of pairwise interaction potentials which correspond to different $\psi_{0}$ values.

At medium $\psi_{0}$ values (e.g. $35 \mathrm{mV}$, highlighted in cyan in Fig. 4), the secondary minimum arises at the potential curve, and particles are held closely together with a characteristic distance of 2-3 nm corresponding to the minimum position. A rather high activation energy for transition between secondary and primary minima prevents irreversible particle aggregation. This might correspond to TEM images related to Phase 2 in Fig. 2. According to the theoretical analysis, the secondary minimum at potential curves exists even in the case of non-charged particles (near-zero $\psi_{0}$ ). However, the activation energy for transition between secondary and primary minima became low $\left(\sim 2 k_{\mathrm{B}} T\right)$. This should lead to irreversible flocculation of the particles. However, this is not the case for a real reaction mixture since the AuNP surface is always covered by either gold chloride complex anions or by citrate/humate anions.

The further growth of $\psi_{0}$ strengthens the repulsion of particle double layers; the secondary minimum shifts to higher $h$ values, becomes shallower, and gradually disappears (compare the curves for 40, 50, and $70 \mathrm{mV}$ highlighted in Fig. 4 in green, yellow, and red, respectively). The thermal motion of particles allows them to abandon this shallow potential well, and they rapidly become redispersed again (compare with Phase 3 in Fig. 2). Conceptual results of calculations are virtually independent on $l$ and $B_{\mathrm{s}}$ values (see the ESI $\dagger$ ). The

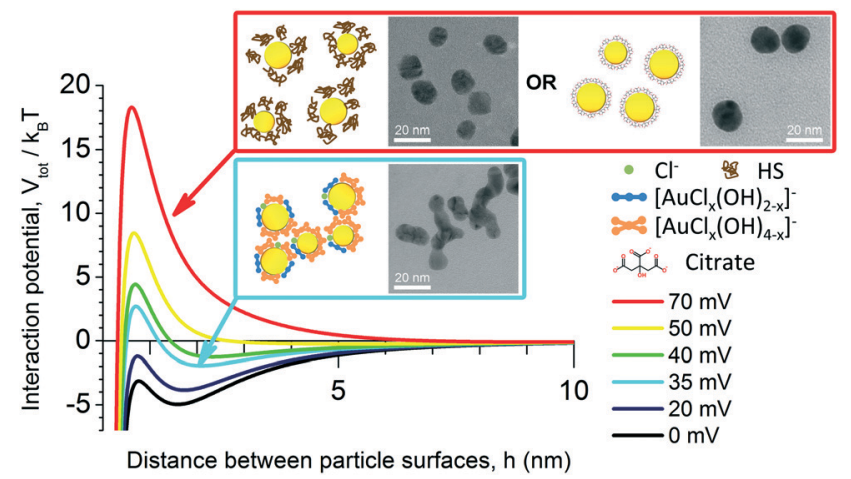

Fig. 4 Pairwise AuNP interaction potentials at different values of surface potential $\psi_{0}$. growing surface potential $\psi_{0}$ has an impact on the particle stability (and pairwise potentials) similar to that in the case of decreasing salt concentration. ${ }^{63}$

Direct calculation of the potential interaction curves in the case of humate-mediated synthesis is more complicated. Firstly, humates are polyelectrolytes of high polydispersity. The exact charge distribution of humate molecules is unknown, which hinders proper calculation of solution ionic strength. Secondly, both electrostatic and solvation terms are valid for the solutions of low-molecular-weight electrolytes, while the behavior of polyelectrolytes in solution is much more complex. Nevertheless, the estimated ionic strength of the humate-based reaction mixture was quite similar to that for the citrate-mediated synthesis. Thus, the qualitative estimates of formation and stabilization of intermediate AuNP aggregates (Fig. 4) ought to be applicable for both citrate and humate-mediated syntheses. Humate sorption on the AuNP surface is enhanced due to multi-site cooperative interactions which contribute to the aggregates' disassembly. These steric effects result in ultra-stable AuNP sols (Fig. S4†).

The above analysis refers to the pairwise interaction of colloidal NPs. In the real system, particle agglomerates with several neighbors are observed. Simple evaluation (similar to the case of liquid-vapor equilibrium) can be done in order to estimate the depth of the secondary minimum needed to make the aggregated state of AuNPs more favorable as compared to the separated one. The equality of chemical potentials of the separated and aggregated particles results in energy gain upon flocculation of about $k_{\mathrm{B}} T \ln \left(n_{\mathrm{agg}} / n_{\text {free }}\right)$ per particle, where $n_{\text {agg }}$ is the NP concentration in agglomerates, and $n_{\text {free }}$ is the concentration of the separated NPs in solution. Since the $n_{\text {agg }} / n_{\text {free }}$ ratio is $\sim 10^{6}$ (roughly estimated from the aggregates' morphology and the presence of some non-aggregated AuNPs even at the early stages of synthesis as observed by TEM (Fig. 2)), we obtain $V_{\min } \sim 14 k_{\mathrm{B}} T$. If the aggregates are large enough and each particle has several neighbors $(n \sim$ 10 ), this value corresponds to a rather mild energy gain of about a few $k_{\mathrm{B}} T$.

The obtained conceptual results are in reasonable agreement with the experimental data. Of particular importance is the appearance of the secondary minimum at the interparticle interaction potential curve at separations of $2-3 \mathrm{~nm}$, providing rationalization for reversible flocculation of AuNPs and intermediate aggregate stabilization with no particle fusion in both humate- and citrate-mediated syntheses. The van der Waals attraction of particles trapped in this minimum is equilibrated both by a repulsion of overlapping electric double layers and a steric repulsion of adsorbed moieties and hydration shells, preventing particles from irreversible flocculation. Simple DLVO calculations without taking into account this steric component would not reveal the existence of the secondary minimum at distances of a few nanometers. At the same time it is the secondary minimum that facilitates stabilization of non-fused particle aggregates producing dendritic and worm-like structures upon drying as observed by TEM. 
The obtained results on the leading role of steric factors in the stabilization of aqueous sols of capped AuNPs provide a reasonable explanation for the water-redispersibility of humate-AuNPs which was examined in the experiments described below.

\section{Water-redispersible properties of humate-stabilized gold nanoparticles}

We have freeze dried stable sols of humate-capped AuHS-7 and citrate-AuNPs. Both sols produced powders, which were redispersed in alkaline aqueous solution upon sonication. The resultant solutions are shown in Fig. 5a and b.

The humate-AuNPs produced a colloidal suspension with typical red color (Fig. 5a), whereas the citrate-AuNPs did not redisperse and gave a gray color to the solution (Fig. 5b). This is indicative of irreversible flocculation of citrate-AuNPs during freeze-drying, which was not the case for the humate-AuNPs. We have measured the UV-vis absorbance spectrum of redispersed humate-AuNPs, obtained their TEM image and compared them to those for the initial sols as shown in Fig. 5c and d. It can be seen that the redispersed humate-AuNPs had the same size distribution and SPR-peak location and intensity as the initial aqueous sol of these AuNPs.

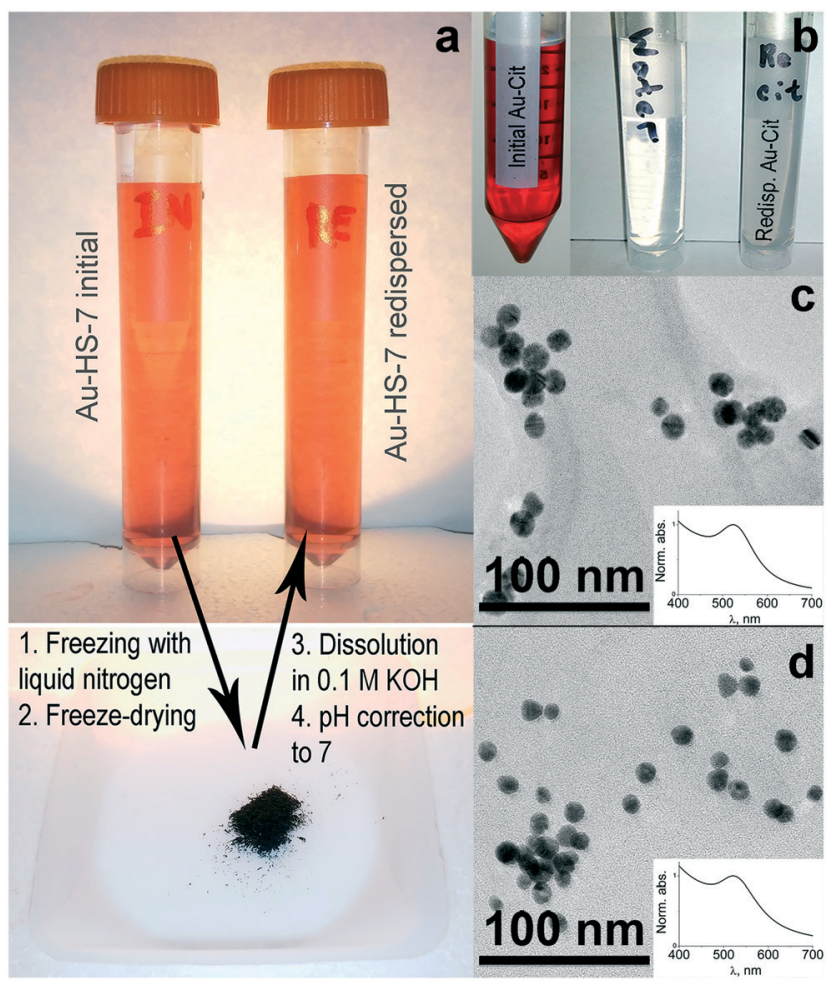

Fig. 5 Redispersion of the humate- and citrate-capped AuNPs in water: (a) humate-capped AuNP sols before and after redispersion and (b) citrate-capped AuNPs before and after redispersion. (c and d) TEM images and SPR peaks of the initial and redispersed humate-capped AuNPs (Au-HS-7 sol), respectively.
The observed difference between humate- and citrateAuNPs could be connected to the polyelectrolytic nature of humate. Macromolecular humate will remain at the metallic surface during freeze-drying, whereas low molecular weight citrate will sublimate. The high hydration affinity of humic macroligands could bring about water-redispersibility of humate-AuNPs.

\section{Conclusions}

The non-classical growth of spheroidal gold nanoparticles (AuNPs) was induced by humates, which are natural hyperbranched polyelectrolytes with carboxylic and phenolic functionalities. Striking similarities between the humate- and the citrate-mediated growth of spheroidal AuNPs have been revealed. Theoretical calculations of pairwise particle interaction potentials revealed the appearance of the secondary minimum at the particle separation of $2-3 \mathrm{~nm}$ which explained the formation of stable intermediate aggregates in the case of both citrate and humate and their follow-up disintegration upon an increase of the charge surface caused by sorption of citrate or humate. These results do not support the recently published opinion of Wuithschick et al. ${ }^{53}$ who referred to citrate-mediated formation of spheroidal monodisperse AuNPs as a "fortunate coincidence created by a favorable interplay of several chemical and physicochemical processes". We believe that in-depth USAXS comparative studies of the citrate and humate mediated growth of spheroidal plasmonic AuNPs, which we plan in the nearest future, might shed new light on the mechanism of AuNP growth mediated by low- and high-molecular weight carboxylic ligands. They will provide more precise tools for directed design of HSbased gold nanomaterials with tailored properties. A unique feature of the proposed synthesis is the formation of waterredispersible AuNPs, which has never been reported for this system before. The polyelectrolyte nature of humate was suggested to be a key factor contributing to strong sorption of humate at the water-metallic gold interface, which enabled water-redispersion of the humate-capped AuNPs. This opens a way to a much wider application of humate-AuNPs in biomedical diagnostics and environmental sensing technologies. To demonstrate the scope of these applications is our immediate goal.

\section{Abbreviations}

$\begin{array}{ll}\text { AuNP } & \text { Gold nanoparticle } \\ \text { CHP } & \begin{array}{l}\text { Coal humate isolated from Powhumus } \\ \text { DM }\end{array} \\ \text { DLVO } & \begin{array}{l}\text { Derjaguin-Landau-Verwey-Overbeek (theory) } \\ \text { FT ICRMS }\end{array} \\ & \begin{array}{l}\text { Fourier transform ion cyclotron resonance mass } \\ \text { spectrometry }\end{array} \\ \text { FWHM } & \text { Full width at half maximum } \\ \text { HRTEM } & \text { High-resolution transmission electron microscopy } \\ \text { HS } & \text { Humic substances } \\ \text { MW } & \text { Molecular weight } \\ \text { NMR } & \text { Nuclear magnetic resonance }\end{array}$




$\begin{array}{ll}\text { NP } & \text { Nanoparticle } \\ \text { pK } & \text { Dissociation constant } \\ \text { PSD } & \text { Particle size distribution } \\ \text { SAXS } & \text { Small-angle X-ray scattering } \\ \text { SEC } & \text { Size exclusion chromatography } \\ \text { SERS } & \text { Surface enhanced Raman scattering } \\ \text { SPI } & \text { Structure Probe, Inc. } \\ \text { SPR } & \text { Surface plasmon resonance } \\ \text { TEM } & \text { Transmission electron microscopy (microscope) } \\ \text { UV-vis } & \text { Ultraviolet-visible (range of electromagnetic } \\ & \text { spectrum) } \\ \text { XRD } & \text { X-ray diffraction }\end{array}$

\section{Acknowledgements}

We would like to thank Dr. Vladimir Volkov (Institute of Crystallography of RAS) and Salim Maduar (Lomonosov MSU) for very useful discussions and suggestions on the manuscript. We express our gratitude to Prof. Eugene Nikolaev (INEPCP RAS) for access to the FTICR MS facilities at the Emanuel Institute of Biochemical Physics of RAS and to Prof. Oleg A. Shlyakhtin (Chem. Dept., MSU) for assistance in freeze-drying experiments. This work was supported by the Russian Science Foundation (project \# 16-14-00167: citrate-mediated synthesis of AuNPs; theoretical calculations of colloidal stability; experiments on water redispersibility of humate-AuNPs) and the Russian Foundation for Basic Research (project \# 13-0401853A: real-time studies of humate-assisted AuNPs formation; characteristics of AuNPs) and the Lomonosov MSU Program of Development (for the use of the Carl Zeiss Libra 200 MC TEM). AYP, AMR and EAS, graduates of the Kolmogorov Advanced Educational Scientific Center of the Lomonosov MSU, thank their school for the strong high-school background.

\section{References}

1 Y.-C. Yeh, B. Creran and V. M. Rotello, Gold nanoparticles: preparation, properties, and applications in bionanotechnology, Nanoscale, 2012, 4, 1871-1880.

2 B. Pelaz, V. Grazu, A. Ibarra, C. Magen, P. del Pino and J. M. de la Fuente, Tailoring the synthesis and heating ability of gold nanoprisms for bioapplications, Langmuir, 2012, 28, 8965-8970.

3 C. N. R. Rao and A. K. Cheetham, Science and technology of nanomaterials: current status and future prospects, J. Mater. Chem., 2001, 11, 2887-2894.

4 M. Brust, M. Walker, D. Bethell, D. J. Schiffrin and R. Whyman, Synthesis of thiol-derivatised gold nanoparticles in a two-phase liquid-liquid system, J. Chem. Soc., Chem. Commun., 1994, 801-802.

5 T. K. Sau and C. J. Murphy, Room temperature, high-yield synthesis of multiple shapes of gold nanoparticles in aqueous solution, J. Am. Chem. Soc., 2004, 126, 8648-8649.

6 B. Nikoobakht and M. A. El-Sayed, Preparation and growth mechanism of gold nanorods (NRS) using seed-mediated growth method, Chem. Mater., 2003, 15, 1957-1962.
7 S. Chen and K. Kimura, Synthesis and characterization of carboxylate-modified gold nanoparticle powders dispersible in water, Langmuir, 1999, 15(4), 1075-1082.

8 C. R. K. Rao, Polyelectrolyte-aided synthesis of gold and platinum nanoparticles: implications in electrocatalysis and sensing, J. Appl. Polym. Sci., 2012, 124(8), 4765-4771.

9 S. Li, Y. Wu, J. Wang, Q. Zhang, Y. Kou and S. Zhang, Double-responsive polyampholyte as a nanoparticle stabilizer: application to reversible dispersion of gold nanoparticles, J. Mater. Chem., 2010, 20, 4379-4384.

10 S. Balasubramanian, S. R. Bezawada and D. Raghavachari, Green, selective, seedless and one-pot synthesis of triangular au nanoplates of controlled size using bael gum and mechanistic study, ACS Sustainable Chem. Eng., 2016, 4(7), 3830-3839.

11 M. N. Nadagouda, N. Iyanna, J. Lalley, C. Han, D. D. Dionysiou and R. S. Varma, Synthesis of silver and gold nanoparticles using antioxidants from blackberry, blueberry, pomegranate, and turmeric extracts, ACS Sustainable Chem. Eng., 2014, 2(7), 1717-1723.

12 M. Annadhasan, T. Muthukumarasamyvel, V. R. Sankar Babu and N. Rajendiran, Green synthesized silver and gold nanoparticles for colorimetric detection of $\mathrm{Hg}^{2+}, \mathrm{Pb}^{2+}$, and $\mathrm{Mn}^{2+}$ in aqueous medium, ACS Sustainable Chem. Eng., 2014, 2(4), 887-896.

13 P. Elia, R. Zach, S. Hazan, S. Kolusheva, Z. Porat and Y. Zeiri, Green Synthesis of gold nanoparticles using plant extracts as reducing agents, Int. J. Nanomed., 2014, 9, 4007-4021.

14 O. V. Kharissova, H. V. Dias, B. I. Kharisov, B. O. Pérez and V. M. Pérez, The greener synthesis of nanoparticles, Trends Biotechnol., 2013, 31(4), 240-248.

15 Y. Park, Y. N. Hong, A. Weyers, Y. S. Kim and R. J. Linhardt, Polysaccharides and phytochemicals: a natural reservoir for the green synthesis of gold and silver nanoparticles, IET Nanobiotechnol., 2011, 5(3), 69-78.

16 Z. Zhong, D. Sim, J. Teo, J. Luo, H. Zhang and A. Gedanken, D-Glucose-derived polymer intermediates as templates for the synthesis of ultrastable and redispersible gold colloids, Langmuir, 2008, 24(9), 4655-4660.

17 D. M. Ozdoba, J. C. Blyth, R. F. Engler, H. Dinel and M. Schnitzer, Leonardite and Humified Organic Matter, in Humic Substances. Structures, Models and Functions, ed. E. A. Ghabbour and G. Davies, RSC, Cambridge, U.K., 2001, pp. 309-313.

18 F. J. Stevenson, Humus Chemistry: Genesis, Composition, Reactions, John Wiley and Sons, Inc., NY, USA, 1994.

19 P. MacCarthy, The principles of humic substances, Soil Sci., 2001, 166, 738-751.

20 M. Klučáková and R. Kolajová, Dissociation Ability of humic acids: spectroscopic determination of $\mathrm{pKa}$ and comparison with multi-step mechanism, React. Funct. Polym., 2014, 78, 1-6.

21 Z. Struyk and G. Sposito, Redox properties of standard humic acids, Geoderma, 2001, 102, 329-346.

22 M. Klučáková and M. Kalina, Composition, Particle size, charge, and colloidal stability of ph-fractionated humic acids, J. Soils Sediments, 2015, 15(9), 1900-1908. 
23 X. Zhang, P. Zhang, Zh. Wu, L. Zhang, G. Zeng and Ch. Zhou, Adsorption of methylene blue onto humic acid-coated $\mathrm{Fe}_{3} \mathrm{O}_{4}$ Nanoparticles, Colloids Surf., A, 2013, 435, 85-90.

24 S. Ghosh, W. Jiang, J. D. McClements and B. Xing, Colloidal stability of magnetic iron oxide nanoparticles: influence of natural organic matter and synthetic polyelectrolytes, Langmuir, 2011, 27(13), 8036-8043.

25 V. K. Sharma, K. M. Siskova, R. Zboril and J. L. GardeaTorresdey, Organic-coated silver nanoparticles in biological and environmental conditions: fate, stability and toxicity, Adv. Colloid Interface Sci., 2014, 204, 15-34.

26 P. Stathi and Y. Deligiannakis, Humic acid-inspired hybrid materials as heavy metal absorbents, J. Colloid Interface Sci., 2010, 351(1), 239-247.

27 E. Illés and E. Tombácz, The effect of humic acid adsorption on $\mathrm{pH}$-dependent surface charging and aggregation of magnetite nanoparticles, J. Colloid Interface Sci., 2006, 295(1), 115-123.

28 M. Delay and F. H. Frimmel, Nanoparticles in aquatic systems, Anal. Bioanal. Chem., 2012, 402(2), 583-592.

29 R. A. Alvarez-Puebla, D. S. dos Santos Jr and R. F. Aroca, SERS detection of environmental pollutants in humic acidgold nanoparticle composite materials, Analyst, 2007, 132, 1210-1214.

30 R. Baigorri, J. M. García-Mina, R. F. Aroca and R. A. AlvarezPuebla, Optical enhancing properties of anisotropic gold nanoplates prepared with different fractions of a natural humic substance, Chem. Mater., 2008, 20, 1516-1521.

31 V. A. Litvin and B. F. Minaev, The size-controllable, one-step synthesis and characterization of gold nanoparticles protected by synthetic humic substances, Mater. Chem. Phys., 2014, 144(1-2), 168-178.

32 A. Yu. Polyakov, A. E. Goldt, T. A. Sorkina, I. V. Perminova, D. A. Pankratov, E. A. Goodilin and Y. D. Tretyakov, Constrained growth of anisotropic magnetic $\delta$-feooh nanoparticles in the presence of humic substances, CrystEngComm, 2012, 14, 8097-8102.

33 A. Yu. Polyakov, T. A. Sorkina, A. E. Goldt, D. A. Pankratov, I. V. Perminova and E. A. Goodilin, Mössbauer spectroscopy of frozen solutions as a stepwise control tool in preparation of biocompatible humic-stabilized feroxyhyte nanoparticles, Hyperfine Interact., 2013, 219(1), 113-120.

34 A. E. Chekanova, T. A. Sorkina, A. L. Dubov, V. N. Nikiforov, G. A. Davidova, I. I. Selezneva, E. A. Goodilin, L. A. Trusov, V. V. Korolev, I. M. Aref'ev, I. V. Perminova and Y. D. Tretyakov, New environmental nontoxic agents for the preparation of core-shell magnetic nanoparticles, Mendeleev Commun., 2009, 19(2), 72-74.

35 T. A. Sorkina, A. Yu. Polyakov, N. A. Kulikova, A. E. Goldt, O. I. Philippova, A. A. Aseeva, A. A. Veligzhanin, Y. V. Zubavichus, D. A. Pankratov, E. A. Goodilin and I. V. Perminova, Natureinspired soluble iron-rich humic compounds: new look at the structure and properties, J. Soils Sediments, 2014, 14(2), 261-268.

36 D. Nečas and P. Klapetek, Gwyddion: an open-source software for SPM data analysis, Cent. Eur. J. Phys., 2012, 10(1), 181-188.
37 T. A. Enache and A. M. Oliveira-Brett, Phenol and parasubstituted phenols electrochemical oxidation pathways, J. Electroanal. Chem., 2011, 655, 9-16.

38 D. V. Goia and E. Matijevic, Tailoring the particle size of monodispersed colloidal gold, Colloids Surf., A, 1999, 146, 139-152.

39 X. Ji, X. Song, J. Li, Y. Bai, W. Yang and X. Peng, Size control of gold nanocrystals in citrate reduction: the third role of citrate, J. Am. Chem. Soc., 2007, 129(45), 13939-13948.

40 J. Turkevich, P. C. Stevenson and J. Hillier, A Study of the nucleation and growth processes in the synthesis of colloidal gold, Discuss. Faraday Soc., 1951, 11, 55-75.

41 G. Frens, Controlled nucleation for the regulation of the particle size in monodisperse gold suspensions, Nature, Phys. Sci., 1973, 241, 20-22.

42 B. Rodríguez-González, P. Mulvaney and L. M. Liz-Marzán, An electrochemical model for gold colloid formation via citrate reduction, Z. Phys. Chem., 2007, 221(3), 415-426.

43 S. Link and M. A. El-Sayed, Size and temperature dependence of the plasmon absorption of colloidal gold nanoparticles, J. Phys. Chem. B, 1999, 103, 4212-4217.

44 D. M. B. P. Milori, L. Martin-Neto, C. Bayer, J. Mielniczuk and V. S. Bagnato, Humification degree of soil humic acids determined by fluorescence spectroscopy, Soil Sci., 2002, 167(11), 739-749.

45 C. H. Gammons, Y. Yu and A. E. Williams-Jones, The disproportionation of gold (I) chloride complexes at 25 to $200{ }^{\circ} \mathrm{C}$, Geochim. Cosmochim. Acta, 1997, 61(10), 1971-1983.

46 S. Kumar, K. S. Gandh and R. Kumar, Modeling of formation of gold nanoparticles by citrate method, Ind. Eng. Chem. Res., 2007, 46(10), 3128-3136.

47 S. Biggs, P. Mulvaney, C. F. Zukoski and F. Grieser, Study of anion adsorption at the gold-aqueous solution interface by atomic force microscopy, J. Am. Chem. Soc., 1994, 116, 9150-9157.

48 J. F. Wall, F. Grieser and C. F. Zukoski, Monitoring chemical reactions at the gold/solution interface using atomic force microscopy, J. Chem. Soc., Faraday Trans., 1997, 93(22), 4017-4020.

49 M. K. Chow and C. F. Zukoski, Gold sol formation mechanisms: role of colloidal stability, J. Colloid Interface Sci., 1994, 165(1), 97-109.

50 B.-K. Pong, H. I. Elim, J.-X. Chong, W. Ji, B. L. Trout and J.-Y. Lee, New insights on the nanoparticle growth mechanism in the citrate reduction of gold(III) salt: formation of the $\mathrm{Au}$ nanowire intermediate and its nonlinear optical properties, J. Phys. Chem. C, 2007, 111, 6281-6287.

51 Y. Mikhlin, A. Karacharov, M. Likhatski, T. Podlipskaya, Y. Zubavichus, A. Veligzhanin and V. Zaikovski, Submicrometer intermediates in the citrate synthesis of gold nanoparticles: new insights into the nucleation and crystal growth mechanisms, J. Colloid Interface Sci., 2011, 362(2), 330-336.

52 J. Polte, T. T. Ahner, F. Delissen, S. Sokolov, F. Emmerling, A. F. Thünemann and R. Kraehnert, Mechanism of gold nanoparticle formation in the classical 
citrate synthesis method derived from coupled in situ XANES and SAXS evaluation, J. Am. Chem. Soc., 2010, 132(4), 1296-1301.

53 M. Wuithschick, A. Birnbaum, S. Witte, M. Sztucki, U. Vainio, N. Pinna, K. Rademann, F. Emmerling, R. Kraehnert and J. Polte, Turkevich in new robes: key questions answered for the most common gold nanoparticle synthesis, ACS Nano, 2015, 9(7), 7052-7071.

54 A. Zherebker, D. Airapetyan, A. I. Konstantinov, Y. I. Kostyukevich, A. S. Kononikhin, I. A. Popov, K. V. Zaitsev, E. N. Nikolaev and I. V. Perminova, Synthesis of model humic substances: a mechanistic study using controllable $\mathrm{H} /$ $\mathrm{D}$ exchange and Fourier transform ion cyclotron resonance mass spectrometry, Analyst, 2015, 140, 4708-4719.

55 Y. Xu and M. Mavrikakis, Adsorption and Dissociation of $\mathrm{O}_{2}$ on Gold Surfaces: Effect of Steps and Strain, J. Phys. Chem. B, 2003, 107, 9298-9307.

56 N. Hanžić, T. Jurkin, A. Maksimović and M. Gotić, The synthesis of gold nanoparticles by a citrate-radiolytical method, Radiat. Phys. Chem., 2015, 106, 77-82.
57 J. N. Israelachvili, Intermolecular and Surface Forces, Academic Press, New York, USA, 2nd edn, 1992.

58 H. C. Hamaker, The London-van der Waals attraction between spherical particles, Physica, 1937, 4(10), 1058-1072.

59 S. Biggs and P. Mulvaney, Measurement of the forces between gold surfaces in water by atomic force microscopy, J. Phys. Chem., 1994, 100, 8501-8505.

$60 \mathrm{H}$. Ohshima, Effective surface potential and double-layer interaction of colloidal particles, J. Colloid Interface Sci., 1995, 174(1), 45-52.

61 K. Lee, A. N. Sathyagal and A. V. McCormickLee, A closer look at an aggregation model of the Stöber process, Colloids Surf., A, 1998, 144, 115-125.

62 T. Kim, K. Lee, M.-S. Gong and S.-W. Joo, Control of gold nanoparticle aggregates by manipulation of interparticle interaction, Langmuir, 2005, 21(21), 9524-9528.

63 D.-H. Tsai, L. F. Pease III, R. A. Zangmeister, M. J. Tarlov and M. R. Zachariah, Aggregation kinetics of colloidal particles measured by gas-phase differential mobility analysis, Langmuir, 2009, 25, 140-146. 